\title{
Chemical and Antioxidant Properties of Wild Edible Mushrooms from Native Nothofagus spp. Forest, Argentina
}

\author{
Carolina V. Toledo ${ }^{1}$, Carolina Barroetaveña ${ }^{1,2,3, *}$, Ângela Fernandes ${ }^{4}$, Lillian Barros ${ }^{4,5}$ and \\ Isabel C. F. R. Ferreira ${ }^{4, *}$ \\ 1 Centro de Investigación y Extensión Forestal Andino Patagónico (CIEFAP), Ruta 259, Km 4, \\ Esquel 9200, Chubut, Argentina; ctoledo@correociefap.org.ar \\ 2 Facultad de Ingeniería, Universidad Nacional de la Patagonia S.J. Bosco, Ruta 259, Km 4, \\ Esquel 9200, Chubut, Argentina \\ 3 Consejo Nacional de Investigaciones Científicas y Tecnológicas (CONICET), Av. Rivadavia 1917, \\ C1033AAJ, CABA, Argentina \\ 4 MountainResearch Centre (CIMO), ESA, Polytechnic Institute of Bragança, Campus de Santa Apolónia, \\ 1172, 5300-253 Bragança, Portugal; afeitor@ipb.pt (A.F.); lillian@ipb.pt (L.B.) \\ 5 Laboratory of Separation and Reaction Engineering-Laboratory of Catalysis and Materials (LSRE-LCM), \\ Polytechnic Institute of Bragança, Campus de Santa Apolónia, 1134, 5301-857 Bragança, Portugal \\ * Correspondence: cbarroetavena@correociefap.org.ar (C.B.); iferreira@ipb.pt (I.C.F.R.F.); \\ Tel.: +54-2945-453-948 (C.B.); +351-273-303-219 (I.C.F.R.F.); \\ Fax: $+54-2945-450-175$ (C.B.); +351-273-325-405 (I.C.F.R.F.)
}

Academic Editor: Derek J. McPhee

Received: 18 August 2016; Accepted: 6 September 2016; Published: 8 September 2016

\begin{abstract}
This study addresses issues regarding chemical and bioactive properties of nine wild edible mushrooms from native Nothofagus forest from Patagonia, Argentina. Macronutrients, sugars, fatty acids, tocopherols, organic acids, phenolic compounds and antioxidant properties were determined. Protein was found in high levels and varied between $3.35 \mathrm{~g} / 100 \mathrm{~g} \mathrm{dw}$ in Cyttaria hariotii and $22.29 \mathrm{~g} / 100 \mathrm{~g} \mathrm{dw}$ in Lepista nuda. All of them presented mannitol and trehalose as main sugars. Mannitol was significantly higher in Ramaria patagonica, although absent in Fistulina endoxantha, whereas trehalose predominated in Aleurodiscus vitellinus, Hydropus dusenii, Cortinarius magellanicus, C. hariotii, Grifola gargal and L. nuda, ranging from 1.15 to $10.26 \mathrm{~g} / 100 \mathrm{~g} \mathrm{dw}$; it was absent in R. patagonica. The major fatty acid found was linoleic acid, followed by oleic acid and palmitic acid. All species presented oxalic and fumaric acids, while some also had malic, quinic and citric acids. Tocopherols composition was variable. Cortinarius magellanicus presented significantly higher contents of both $\alpha$-tocopherol and $\beta$-tocopherol. R. patagonica presented the best results in all the antioxidant activity assays ( $\mathrm{EC}_{50}$ values $\leq 1 \mathrm{mg} / \mathrm{mL}$ ) and the highest content of phenolic compounds presenting gallic, $p$-hydroxybenzoic, $p$-coumaric and cinnamic acids. This study constitutes the first report on chemical composition and nutritional value of most of these edible mushroom species. Furthermore, it provides important information necessary to characterize and define the use of these species as gastronomic delicacies, functional foods and sources of bioactive compounds.
\end{abstract}

Keywords: macrofungi; nutrients; nutritional value; phenolic compounds; antioxidant

\section{Introduction}

The consumption of wild edible mushrooms in cooking, traditional medicine and religious rituals is a practice that dates back to the beginnings of civilization [1] and has been developed and maintained in different countries around the world, particularly China in Asia [2-4], Spain and Italy in Europe [1] and Mexico in Latin America [5]. 
Wild mushrooms are becoming more and more important in our diet for their nutritional value, including high protein, vitamins and minerals and low fat/energy contents [6-9]. The fatty acids composition may also have beneficial effects on blood lipid profiles. Substitution of saturated fatty acids (SFAs) with monounsaturated fatty acids (MUFAs) leads to increased high density lipoprotein (HDL) cholesterol and decreased low-density lipoprotein (LDL) cholesterol, triacylglycerols, lipid oxidation and LDL susceptibility to oxidation [10]. The study of edible fungi in various parts of the world has included the analysis of the chemical composition and nutritional values as elements necessary to characterize and define other uses besides the gastronomic, as reported for wild mushrooms and cultivated in Spain [11], Italy [12], Portugal [13,14], Greece [15,16], Turkey [17], India [18], Iran [19], Thailand [20], Serbia [21], Brazil [22], Mexico [23] and Argentine [24-26]. In recent years, increased interest in human health, nutrition and disease prevention has enlarged consumer demand for functional foods [27-29]. In fact, mushrooms have become attractive as functional foods and as a source of bioactive compounds [30,31]. Some species are rich sources of antioxidant compounds such as phenolic compounds and tocopherols [32-38].

Nothofagus spp. forests (in Patagonian Andes region, Argentina) harbor several fungal species $[39,40]$ with edible potential and high nutritional value. In particular, the species Aleurodiscus vitellinus (Lev.) Pat., Cortinarius magellanicus Speg., Hydropus dusenii (Bres.) Singer., Cyttaria hariotii E. Fisch. Fistulina antarctica Speg., F. endoxantha Speg., Grifola gargal Singer, Lepista nuda (Bull.) Cooke. and Ramaria patagonica (Speg.) Corner, have records of consumption. However, background information on their chemical composition and bioactive properties is very scarce and restricted to C. hariotii [41,42], G. gargal [43-48] and L. nuda [13,15,49,50].

Knowledge on chemical composition allows the use of wild fungi as food or bioactive resources and favors regional economies related to non-wood forest products harvesting, gastronomy and health care. Diverse uses of these resources would promote better management and conservation actions on the habitat where they grow.

This study addresses issues regarding chemical and bioactive properties of wild edible mushrooms from native Nothofagus forest, in order to evaluate them as sources of nutrients and bioactive compounds. Chemical analysis included determination of macronutrients and individual profiles in sugars, fatty acids and organic acids. The bioactivity evaluation was focused on the analysis of phenolic compounds (total and individuals) and antioxidant properties (free radicals scavenging activity, reducing power and inhibition of lipid peroxidation).

\section{Results and Discussion}

\subsection{Nutritional Value}

The results of the composition in terms of nutrients and estimated energetic value (expressed in dry weight basis) obtained for the nine wild edible fungal species are shown in Table 1.

Protein was found in high levels and varied between $3.35 \mathrm{~g} / 100 \mathrm{~g} \mathrm{dw}$ in C. hariotii and $22.29 \mathrm{~g} / 100 \mathrm{~g} \mathrm{dw}$ in L. nuda. The highest protein content observed in L. nuda was within the range of previous studies, that reported values between 19.8 and $59.4 \mathrm{~g} / 100 \mathrm{~g} \mathrm{dw}$ [13,15,49]. Comparing R. patagonica with other Ramaria species, R. flava gave higher values (35.55 g/100 g dw) [51], as also $R$. botrytis (39.8 g/100 $\mathrm{g} \mathrm{dw}$ ) [13]. C. magellanicus and H. duseni, within a range of 13 to $16 \mathrm{~g} / 100 \mathrm{~g} \mathrm{dw}$ of protein content, showed similar values to those reported for Suillus granulatus (L.) Roussel (16.5 g/100 g dw) and Cortinarius praestans Cordier (14.54 g/100 g dw) by Ouzouni and Riganakos [15] and Pereira et al. [52], respectively. G. gargal, A. vitellinus and F. endoxantha yielded values between 5 to $8 \mathrm{~g} / 100 \mathrm{~g}$ dw. Previous studies on G. gargal reported similar results to those found in this one [43]. F. antarctica and C. hariotii showed the lowest values, between 3 to $4 \mathrm{~g} / 100 \mathrm{~g} \mathrm{dw}$ protein content, the former showing lower values $(7.50 \mathrm{~g} / 100 \mathrm{~g} \mathrm{dw})$ to those obtained by Schmeda-Hirschmann et al. [42]. Other studies with different species of Fistulina have reported higher values $(63.69 \mathrm{~g} / 100 \mathrm{~g})$ [53] than those reported here for F. antarctica and F. endoxantha. Protein 
content of mushroom species could be affected by a number of factors, namely the developmental stage, the sampled part, the level of available nitrogen and location [54]. Furthermore, the conversion factor used to calculate the protein content can influence its outcome $[8,55]$.

Crude fat content ranged from $0.83 \mathrm{~g} / 100 \mathrm{~g}$ dw in F. antarctica and $4.29 \mathrm{~g} / 100 \mathrm{~g} \mathrm{dw}$ in H. dusenii. Previous reports of C. hariotii and G. gargal fat content were higher than those reported here (5 and $7.5 \mathrm{~g} / 100 \mathrm{~g} \mathrm{dw}$, respectively) [42,43]. L. nuda fat content was close to that obtained by Colak et al. [49] for the same species $(9.04 \mathrm{~g} / 100 \mathrm{~g} \mathrm{dw})$. Carbohydrates were also an abundant macronutrient and ranging from $67.58 \mathrm{~g} / 100 \mathrm{~g} \mathrm{dw}$ in C. magellanicus to $94.22 \mathrm{~g} / 100 \mathrm{~g} \mathrm{dw}$ in F. antarctica. Comparisons with previous reports showed lower values for C. hariotii (75 g/100 g dw) [42] and similar for G. gargal [43] and L. nuda [15].

Ash content was also variable between species and ranged from $1.24 \mathrm{~g} / 100 \mathrm{~g}$ in F. antarctica to $13.92 \mathrm{~g} / 100 \mathrm{~g}$ in C. magellanicus. The parasitic C. hariotii yielded ash values lower (7.0 g/100 g dw) than what have been previously reported by Schmeda-Hirschmann et al. [42], whereas L. nuda showed higher values (5.4 and $6.0 \mathrm{~g} / 100 \mathrm{~g} \mathrm{dw}$ ) than those reported by Colak et al. [49] and Ouzouni \& Riganakos [15] respectively and lower values $(18.5 \mathrm{~g} / 100 \mathrm{~g} \mathrm{dw})$ than the reported by Barros et al. [13]. G. gargal yielded lower values than what reported by Schmeda-Hirschmann et al. [43] for the same species $(5.0 \mathrm{~g} / 100 \mathrm{~g} \mathrm{dw})$.

The highest value of energetic contribution was observed in F. antarctica, while C. magellanicus presented the lowest value (Table 1). The energy contribution of the different species under study was calculated on the basis of nutritional analysis (Table 1); the highest values were observed in F. antarctica (399.18 kcal/100 $\mathrm{g} \mathrm{dw})$, while C. magellanicus presented the lowest value (358.03 kcal/100 $\mathrm{g} \mathrm{dw})$.

Table 1. Proximate chemical composition (g/100 g) and energetic value (kcal/100 g) of the studied wild mushrooms on a dry weight basis (mean $\pm \mathrm{SD}$ ).

\begin{tabular}{|c|c|c|c|c|c|}
\hline Species & Total Fat & Crude Protein & Carbohydrates & Ash & Energy \\
\hline A. vitellinus & $3.49 \pm 0.20 \mathrm{~b}$ & $7.38 \pm 0.21 \mathrm{e}$ & $84.23 \pm 0.32 d$ & $4.90 \pm 0.05 \mathrm{e}$ & $397.84 \pm 0.56 \mathrm{ab}$ \\
\hline H. dusenii & $4.29 \pm 0.22 \mathrm{a}$ & $13.52 \pm 0.43 \mathrm{~d}$ & $75.96 \pm 0.86 \mathrm{~g}$ & $6.23 \pm 0.45 \mathrm{~d}$ & $396.53 \pm 1.99 \mathrm{abc}$ \\
\hline C. magellanicus & $2.75 \pm 0.12 c$ & $15.75 \pm 0.10 c$ & $67.58 \pm 0.27 i$ & $13.92 \pm 0.17 \mathrm{a}$ & $358.03 \pm 0.08 \mathrm{~g}$ \\
\hline C. hariotii & $1.31 \pm 0.03 \mathrm{de}$ & $3.35 \pm 0.15 \mathrm{~g}$ & $92.51 \pm 0.25 b$ & $2.83 \pm 0.18 \mathrm{~g}$ & $395.24 \pm 0.39 b c$ \\
\hline F. antarctica & $0.83 \pm 0.01 \mathrm{e}$ & $3.71 \pm 0.07 \mathrm{~g}$ & $94.22 \pm 0.07 a$ & $1.24 \pm 0.01 \mathrm{~h}$ & $399.18 \pm 0.06 a$ \\
\hline F. endoxantha & $1.19 \pm 0.03 \mathrm{e}$ & $7.76 \pm 0.33 e^{\circ}$ & $79.84 \pm 0.58 f$ & $11.21 \pm 0.55 b$ & $361.09 \pm 1.43 \mathrm{f}$ \\
\hline G. gargal & $1.79 \pm 0.09 \mathrm{~d}$ & $5.96 \pm 0.49 f$ & $88.59 \pm 0.53 c$ & $3.66 \pm 0.28 f$ & $394.33 \pm 1.12 c$ \\
\hline L. nuda & $0.84 \pm 0.02 \mathrm{e}$ & $22.29 \pm 0.44 \mathrm{a}$ & $68.29 \pm 0.66 \mathrm{hi}$ & $8.58 \pm 0.53 c$ & $369.84 \pm 1.58 \mathrm{e}$ \\
\hline R. patagonica & $2.51 \pm 0.10 c$ & $19.68 \pm 0.64 b$ & $69.34 \pm 0.53 h$ & $8.47 \pm 0.25 c$ & $378.67 \pm 0.71 d$ \\
\hline Homoscedasticity $^{1}$ & 0.027 & 0.288 & 0.036 & 0.256 & 0.199 \\
\hline 1-way ANOVA 2 & $<0.001$ & $<0.001$ & $<0.001$ & $<0.001$ & $<0.001$ \\
\hline
\end{tabular}

${ }^{1}$ Homoscedasticity among mushroom samples was tested by the Levene test: homoscedasticity, $p>0.05$; heteroscedasticity, $p<0.05 .{ }^{2} p<0.05$ indicates that the mean value of at least one component differs from the others (in this case, multiple comparison tests were performed). $p$-Value $(n=81)$. For each mushroom sample, means within a column with different letters differ significantly $(p<0.05)$.

Concerning sugars composition (Table 2), the nine mushrooms showed some homogeneity. All of them presented mannitol and trehalose as main sugars, as has been previously reported for other wild mushroom species [13,35,52]. Mannitol content was significantly higher for $R$. patagonica, although absent in F. endoxantha, whereas trehalose predominated in A. vitellinus, H. dusenii, C. magellanicus, C. hariotii, G. gargal and L. nuda, ranging from 1.15 to $10.26 \mathrm{~g} / 100 \mathrm{~g} \mathrm{dw}$ and was absent in R. patagonica. Other sugars such as fructose and rhamnose were present in lower abundance in some of the studied species, but predominated in F. endoxantha and F. antarctica, respectively. Sucrose was only detected in H. dusenii. In terms of total sugars content, F. antarctica revealed the highest value (28.68 g/100 g dw), while C. hariotii the lowest $(4.02 \mathrm{~g} / 100 \mathrm{~g} \mathrm{dw})$. This is the first report of sugar profiles for the species included in this study, except L. nuda, which has been described by Colak et al. [13,15,49]. A. vitellinus and C. hariotii presented similar quantities of trehalose than Agaricus lutosus (Møller) Møller and Leucoagaricus leucothites (Vittad.) Wasser, respectively [52]. Meanwhile, mannitol values 
obtained for H. dusenii and R. patagonica were similar to the ones reported by Barros et al. [35] for Cantharellus cibarius L. ex Fr. and Marasmius oreades (Bolt. ex Fr.) Fr., respectively. Mannitol and trehalose concentrations on dry specimens of L. nuda were different $(0.80 \mathrm{~g} / 100 \mathrm{~g}$ and $12.03 \mathrm{~g} / 100 \mathrm{~g} \mathrm{dw}$, respectively) from those reported by Barros et al. [35].

Table 2. Sugars composition ( $\mathrm{g} / 100 \mathrm{~g}$ on dry weight basis) of the studied wild mushrooms (mean $\pm \mathrm{SD}$ ).

\begin{tabular}{|c|c|c|c|c|c|c|}
\hline Species & Rhamnose & Fructose & Mannitol & Trehalosa & Sucrose & Total Sugars \\
\hline A. vitellinus & $0.58 \pm 0.07 c$ & $0.67 \pm 0.03 c$ & $1.44 \pm 0.10 \mathrm{~d}$ & $3.38 \pm 0.04 \mathrm{~d}$ & nd & $6.07 \pm 0.24 f$ \\
\hline H. dusenii & nd & nd & $2.57 \pm 0.12 c$ & $10.26 \pm 0.01 a$ & $1.25 \pm 0.15$ & $14.08 \pm 0.28 \mathrm{c}$ \\
\hline C. magellanicus & nd & nd & $3.92 \pm 0.16 b$ & $5.00 \pm 0.03 c$ & nd & $8.92 \pm 0.14 \mathrm{e}$ \\
\hline C. hariotii & nd & nd & $0.81 \pm 0.01 \mathrm{e}$ & $3.21 \pm 0.02 \mathrm{e}$ & nd & $4.02 \pm 0.03 \mathrm{~g}$ \\
\hline F. antarctica & $13.18 \pm 0.32 \mathrm{a}$ & $11.65 \pm 0.37 a$ & $1.26 \pm 0.20 \mathrm{~d}$ & $2.59 \pm 0.09 f$ & nd & $28.68 \pm 0.58 \mathrm{a}$ \\
\hline F. endoxantha & $6.09 \pm 0.68 b$ & $9.92 \pm 0.01 b$ & nd & $2.04 \pm 0.02 \mathrm{~g}$ & nd & $18.05 \pm 0.65 b$ \\
\hline G. gargal & nd & $0.15 \pm 0.02 \mathrm{e}$ & $0.47 \pm 0.11 \mathrm{f}$ & $1.15 \pm 0.07 \mathrm{~h}$ & nd & $1.77 \pm 0.16 h$ \\
\hline L. nuda & nd & $0.29 \pm 0.02 \mathrm{~d}$ & $3.64 \pm 0.34 b$ & $6.23 \pm 0.17 b$ & nd & $10.16 \pm 0.49 \mathrm{~d}$ \\
\hline R. patagonica & nd & $0.29 \pm 0.03 \mathrm{~d}$ & $9.12 \pm 0.32 \mathrm{a}$ & nd & nd & $9.41 \pm 0.35 \mathrm{e}$ \\
\hline Homoscedasticity $^{1}$ & 0.012 & 0.009 & 0.175 & 0.054 & 0.005 & 0.317 \\
\hline 1-way ANOVA 2 & $<0.001$ & $<0.001$ & $<0.001$ & $<0.001$ & $<0.001$ & $<0.001$ \\
\hline
\end{tabular}

${ }^{1}$ Homoscedasticity among mushroom samples was tested by the Levene test: homoscedasticity, $p>0.05$; heteroscedasticity, $p<0.05 .^{2} p<0.05$ indicates that the mean value of at least one component differs from the others (in this case, multiple comparison tests were performed). $p$-Value $(n=81)$. For each mushroom sample, means within a column with different letters differ significantly $(p<0.05)$. nd $=$ not detected.

The results of fatty acids composition, total saturated fatty acids (SFA), monounsaturated fatty acids (MUFA) and polyunsaturated fatty acids (PUFA) of the studied mushrooms are shown in Table 3. In general, the major fatty acid found in the studied species was linoleic acid (C18:2), followed by oleic acid (C18:1) and palmitic acid (C16:0). Besides the three main fatty acids already described, 21 more were identified and quantified. PUFA were the main group of fatty acids, significantly higher than MUFA and SFA in H. dusenii, F. antarctica, F. endoxantha, R. patagonica, C. hariotii, C. magellanicus and $L$. nuda, the latter with the highest value due to the high contribution of linoleic acid $(72 \%)$. In A. vitellinus and G. gargal, MUFA were the main group, due to the high contribution of oleic acid, ranging from $45 \%$ to $52 \%$. UFA (unsaturated fatty acids) predominated over SFA in all the studied species, ranging from $64.1 \%$ and $83.40 \%$. The main fatty acids found in this study were linoleic acid, followed by oleic acid and palmitic acid, coincidently with that observed for species of mushrooms at different studied here [52,56-59]. Oleic acid is a monounsaturated fatty acid omega 9 series typical of vegetable oils such as olive oil and is known for its effectiveness in reducing cholesterol levels [60], which promotes the decrease of cardiovascular diseases [61]. Linoleic acid is an essential fatty acid as it cannot be synthesized by the human organism, due to the lack of desaturase enzymes required for its production [62].

Regarding the organic acids composition, all species presented oxalic and fumaric acids; some species also revealed the presence of malic, quinic and citric acids (Table 4). The oxalic acid content revealed significant differences among species, being higher for $L$. nuda and R. patagonica although most species showed lower concentrations, between 0.10 to $0.54 \mathrm{~g} / 100 \mathrm{~g}$ dw. Fumaric acid, with antioxidant, antimicrobial and acidifying properties [63], was higher for C. magellanicus, while A. vitellinus and C. hariotti presented the lowest values. Malic acid was found in five of the nine studied species, C. magellanicus with the highest content and C. hariotti with the lowest content. Quinic acid was only detected in F. antarctica and L. nuda while citric acid, important in the prevention of mushrooms browning [63] was only present in C. magellanicus and R. patagonica. The total organic acids content ranged from $0.21 \mathrm{~g} / 100 \mathrm{~g} \mathrm{dw}$ in A. vitellinus to $28.87 \mathrm{~g} / 100 \mathrm{~g} \mathrm{dw}$ in L. nuda. This is the first report on organic acids composition for all the studied species, except $L$. nuda, whose previous reported values were higher (4.34 g/100 g) [64] than those found in this work. 
Table 3. Fatty acids composition (relative percentage) of the studied wild mushrooms (mean \pm SD).

\begin{tabular}{|c|c|c|c|c|c|c|c|c|c|c|c|}
\hline & A. vitellinus & H. dusenii & $\begin{array}{c}C . \\
\text { magellanicus }\end{array}$ & C. hariotii & F. antarctica & F. endoxantha & G. gargal & L. nuda & R. patagonica & Homoscedasticity $^{1}$ & $\begin{array}{c}\text { 1-Way } \\
\text { ANOVA }^{2}\end{array}$ \\
\hline C6:0 & $0.06 \pm 0.01 \mathrm{c}$ & $0.55 \pm 0.06 a$ & $0.25 \pm 0.01 \mathrm{~b}$ & $0.06 \pm 0.01 c$ & $0.18 \pm 0.01 \mathrm{~b}$ & $0.20 \pm 0.02 b$ & $0.07 \pm 0.01 c$ & $0.20 \pm 0.01 b$ & $0.081 \pm 0.001 \mathrm{c}$ & 0.188 & $<0.001$ \\
\hline C8:0 & $0.04 \pm 0.01 \mathrm{~cd}$ & $0.12 \pm 0.01 \mathrm{~b}$ & $0.05 \pm 0.01 \mathrm{c}$ & $0.11 \pm 0.02 b$ & $0.10 \pm 0.01 \mathrm{~b}$ & $0.20 \pm 0.02 \mathrm{a}$ & $0.05 \pm 0.00 \mathrm{c}$ & $0.06 \pm 0.01 c$ & $0.03 \pm 0.01 \mathrm{~d}$ & 0.516 & $<0.001$ \\
\hline C10:0 & $0.031 \pm 0.001 \mathrm{~d}$ & $0.11 \pm 0.01 \mathrm{c}$ & $0.029 \pm 0.001 \mathrm{~d}$ & $0.09 \pm 0.01 \mathrm{c}$ & $0.17 \pm 0.03 \mathrm{~b}$ & $0.42 \pm 0.04 a$ & $0.029 \pm 0.001 \mathrm{~d}$ & $0.041 \pm 0.001 \mathrm{~d}$ & $0.019 \pm 0.001 \mathrm{~d}$ & 0.057 & $<0.001$ \\
\hline C12:0 & $0.039 \pm 0.001 \mathrm{e}$ & $0.11 \pm 0.01 d$ & $0.049 \pm 0.001 \mathrm{e}$ & $0.19 \pm 0.01 \mathrm{c}$ & $0.22 \pm 0.04 \mathrm{~b}$ & $0.55 \pm 0.02 a$ & $0.091 \pm 0.001 \mathrm{~d}$ & $0.090 \pm 0.001 \mathrm{~d}$ & $0.031 \pm 0.001 \mathrm{e}$ & 0.049 & $<0.001$ \\
\hline C14:0 & $0.33 \pm 0.05 \mathrm{f}$ & $0.60 \pm 0.01 \mathrm{~cd}$ & $0.22 \pm 0.03 \mathrm{f}$ & $0.66 \pm 0.04 c$ & $1.57 \pm 0.07 \mathrm{~b}$ & $2.43 \pm 0.03 a$ & $0.25 \pm 0.04 \mathrm{f}$ & $0.44 \pm 0.02 \mathrm{e}$ & $0.53 \pm 0.08 \mathrm{de}$ & 0.122 & $<0.001$ \\
\hline C14:1 & $0.04 \pm 0.01 \mathrm{c}$ & $0.051 \pm 0.001 \mathrm{c}$ & $0.011 \pm 0.001 \mathrm{~d}$ & $0.05 \pm 0.01 \mathrm{c}$ & $0.10 \pm 0.01 \mathrm{~b}$ & $0.15 \pm 0.01 \mathrm{a}$ & $0.13 \pm 0.01 \mathrm{ab}$ & nd & nd & 0.038 & $<0.001$ \\
\hline C15:0 & $0.75 \pm 0.06 \mathrm{~b}$ & $0.57 \pm 0.01 \mathrm{c}$ & $0.57 \pm 0.03 \mathrm{c}$ & $0.21 \pm 0.02 \mathrm{e}$ & $0.57 \pm 0.04 \mathrm{c}$ & $0.57 \pm 0.01 \mathrm{c}$ & $0.38 \pm 0.04 \mathrm{~d}$ & $0.26 \pm 0.03 \mathrm{e}$ & $1.08 \pm 0.08 \mathrm{a}$ & 0.051 & $<0.001$ \\
\hline C16:0 & $18.25 \pm 0.86 c$ & $14.46 \pm 0.28 \mathrm{e}$ & $19.21 \pm 0.70 \mathrm{~b}$ & $18.23 \pm 0.24 \mathrm{c}$ & $16.62 \pm 0.79 \mathrm{~d}$ & $20.17 \pm 0.12 \mathrm{a}$ & $17.44 \pm 0.46 \mathrm{~cd}$ & $12.45 \pm 0.03 \mathrm{~g}$ & $13.44 \pm 0.42 \mathrm{f}$ & 0.297 & $<0.001$ \\
\hline C16:1 & $1.45 \pm 0.16 \mathrm{~b}$ & $0.43 \pm 0.01 \mathrm{f}$ & $0.38 \pm 0.02 \mathrm{fg}$ & $0.70 \pm 0.04 \mathrm{~d}$ & $1.02 \pm 0.08 \mathrm{c}$ & $1.84 \pm 0.01 \mathrm{a}$ & $0.61 \pm 0.01 \mathrm{~d}$ & $0.46 \pm 0.02 \mathrm{f}$ & $0.28 \pm 0.08 \mathrm{~g}$ & 0.060 & $<0.001$ \\
\hline C17:0 & $0.13 \pm 0.01 \mathrm{e}$ & $0.22 \pm 0.01 \mathrm{de}$ & $0.10 \pm 0.01 \mathrm{e}$ & $0.34 \pm 0.03 \mathrm{~cd}$ & $0.68 \pm 0.08 \mathrm{~b}$ & $0.39 \pm 0.04 c$ & $0.13 \pm 0.01 \mathrm{e}$ & $0.15 \pm 0.01 \mathrm{e}$ & $1.16 \pm 0.08 \mathrm{a}$ & 0.012 & $<0.001$ \\
\hline C18:0 & $3.69 \pm 0.03 c$ & $3.95 \pm 0.18 c$ & $3.30 \pm 0.07 \mathrm{~d}$ & $3.72 \pm 0.20 c$ & $7.37 \pm 0.04 a$ & $4.52 \pm 0.40 \mathrm{~b}$ & $7.31 \pm 0.06 a$ & $1.95 \pm 0.04 \mathrm{e}$ & $3.84 \pm 0.01 \mathrm{c}$ & 0.032 & $<0.001$ \\
\hline C18:1n 9 & $52.08 \pm 0.45 a$ & $33.73 \pm 2.03 c$ & $9.04 \pm 0.15 \mathrm{gf}$ & $7.93 \pm 0.80 \mathrm{f}$ & $26.63 \pm 0.02 \mathrm{e}$ & $23.53 \pm 0.01 \mathrm{f}$ & $45.28 \pm 0.03 \mathrm{~b}$ & $9.81 \pm 0.22 \mathrm{~g}$ & $29.58 \pm 0.67 \mathrm{~d}$ & 0.038 & $<0.001$ \\
\hline $\mathrm{C} 18: 2 \mathrm{n} 6$ & $19.28 \pm 0.60 \mathrm{~g}$ & $42.69 \pm 1.69 \mathrm{~d}$ & $63.75 \pm 0.69 \mathrm{~b}$ & $34.68 \pm 0.29 \mathrm{e}$ & $42.97 \pm 1.13 \mathrm{~d}$ & $43.63 \pm 0.85 \mathrm{~d}$ & $26.55 \pm 0.03 f$ & $71.32 \pm 0.24 \mathrm{a}$ & $45.35 \pm 0.69 c$ & 0.352 & $<0.001$ \\
\hline C18:3n3 & $2.02 \pm 0.16 \mathrm{~b}$ & $0.10 \pm 0.01 \mathrm{~d}$ & $0.10 \pm 0.01 \mathrm{~d}$ & $11.56 \pm 0.40 \mathrm{a}$ & $0.24 \pm 0.06 \mathrm{~cd}$ & $0.13 \pm 0.03 \mathrm{~cd}$ & $0.25 \pm 0.01 \mathrm{~cd}$ & $0.39 \pm 0.01 \mathrm{c}$ & $0.07 \pm 0.01 \mathrm{~d}$ & 0.019 & $<0.001$ \\
\hline C $20: 0$ & $0.29 \pm 0.01 \mathrm{c}$ & $0.07 \pm 0.01 \mathrm{f}$ & $0.22 \pm 0.01 \mathrm{~d}$ & $2.70 \pm 0.08 a$ & $0.16 \pm 0.02 \mathrm{de}$ & $0.10 \pm 0.01 \mathrm{ef}$ & $0.15 \pm 0.01 \mathrm{e}$ & $0.11 \pm 0.01 \mathrm{ef}$ & $0.59 \pm 0.07 \mathrm{~b}$ & 0.049 & $<0.001$ \\
\hline C20:1 & $0.17 \pm 0.01 \mathrm{~b}$ & $0.10 \pm 0.03 c$ & $0.06 \pm 0.01 \mathrm{~d}$ & $0.07 \pm 0.01 \mathrm{~d}$ & $\begin{array}{c}0.10 \pm 0.01 \mathrm{c} \\
0.10 \pm 0.02\end{array}$ & $0.07 \pm 0.01 \mathrm{~d}$ & $0.16 \pm 0.02 \mathrm{~b}$ & $\begin{array}{l}\text { nd } \\
\text { nd }\end{array}$ & $0.42 \pm 0.01 \mathrm{a}$ & 0.301 & $<0.001$ \\
\hline C20:2 & $\begin{array}{l}0.17 \pm 0.010 \\
0.001 \mathrm{f}\end{array}$ & $0.10 \pm 0.001 \mathrm{ef}$ & $\begin{array}{l}0.06 \pm 0.01 \mathrm{a} \\
0.09 \pm 0.01 \mathrm{~d}\end{array}$ & $1.08 \pm 0.04 a$ & $0.041 \pm 0.001 \mathrm{ef}$ & $0.08 \pm 0.01 \mathrm{de}$ & $\begin{array}{c}0.16 \pm 0.00 \mathrm{D} \\
0.052 \pm 0.001 \mathrm{de}\end{array}$ & $0.14 \pm 0.01 \mathrm{c}$ & $\begin{array}{l}0.42 \pm 0.01 \mathrm{a} \\
0.59 \pm 0.04 \mathrm{~b}\end{array}$ & 0.184 & $<0.001$ \\
\hline $\mathrm{C} 20: 3 \mathrm{n} 3+\mathrm{C} 21: 0$ & $0.042 \pm 0.001 \mathrm{c}$ & $0.15 \pm 0.03 \mathrm{abc}$ & $0.34 \pm 0.04 \mathrm{a}$ & $0.25 \pm 0.02 \mathrm{abc}$ & $0.071 \pm 0.001 \mathrm{bc}$ & $0.23 \pm 0.05 \mathrm{abc}$ & $0.041 \pm 0.001 \mathrm{c}$ & $0.29 \pm 0.01 \mathrm{abc}$ & $0.31 \pm 0.04 \mathrm{ab}$ & 0.055 & $<0.001$ \\
\hline C20:5n 3 & $0.043 \pm 0.002 \mathrm{~d}$ & $0.17 \pm 0.04 \mathrm{~b}$ & $0.52 \pm 0.05 a$ & nd & $0.051 \pm 0.001 \mathrm{~d}$ & nd & $0.09 \pm 0.01 \mathrm{c}$ & $0.55 \pm 0.02 a$ & nd & 0.074 & $<0.001$ \\
\hline C22:0 & $0.45 \pm 0.03 \mathrm{~d}$ & $0.17 \pm 0.01 \mathrm{~g}$ & $0.91 \pm 0.09 \mathrm{c}$ & $3.84 \pm 0.05 a$ & $0.38 \pm 0.07 \mathrm{e}$ & $0.25 \pm 0.01 \mathrm{f}$ & $0.32 \pm 0.05 \mathrm{ef}$ & $0.29 \pm 0.05 \mathrm{f}$ & $1.01 \pm 0.02 \mathrm{~b}$ & 0.129 & $<0.001$ \\
\hline $\mathrm{C} 22: \ln 9$ & $0.08 \pm 0.01 \mathrm{~b}$ & $0.062 \pm 0.001 \mathrm{bc}$ & $0.11 \pm 0.01 \mathrm{~b}$ & $0.21 \pm 0.01 \mathrm{a}$ & nd & $0.051 \pm 0.002 \mathrm{c}$ & nd & $0.21 \pm 0.01 \mathrm{a}$ & $0.091 \pm 0.005 \mathrm{~b}$ & 0.040 & $<0.001$ \\
\hline C23:0 & $0.062 \pm 0.001 \mathrm{~b}$ & nd & $0.043 \pm 0.001 \mathrm{c}$ & nd & $0.041 \pm 0.004 \mathrm{c}$ & $0.028 \pm 0.001 \mathrm{c}$ & nd & nd & $0.45 \pm 0.03 a$ & 0.021 & $<0.001$ \\
\hline C24:0 & $0.55 \pm 0.03 c$ & $0.46 \pm 0.02 c$ & $0.17 \pm 0.02 \mathrm{e}$ & $5.71 \pm 0.22 a$ & $0.52 \pm 0.02 c$ & $0.32 \pm 0.03 \mathrm{~d}$ & $0.53 \pm 0.04 c$ & $0.54 \pm 0.08 c$ & $0.94 \pm 0.03 b$ & 0.041 & $<0.001$ \\
\hline C24:1 & $0.09 \pm 0.01 \mathrm{e}$ & $1.07 \pm 0.05 \mathrm{~b}$ & $0.49 \pm 0.02 c$ & $7.61 \pm 0.13 a$ & $0.21 \pm 0.02 \mathrm{de}$ & $0.13 \pm 0.02 \mathrm{de}$ & $0.09 \pm 0.01 \mathrm{e}$ & $0.23 \pm 0.02 \mathrm{~d}$ & $0.11 \pm 0.05 \mathrm{de}$ & 0.076 & $<0.001$ \\
\hline Total SFA & $24.67 \pm 1.01 \mathrm{e}$ & $21.40 \pm 0.03 \mathrm{~g}$ & $25.12 \pm 0.71 \mathrm{e}$ & $35.85 \pm 0.47 \mathrm{a}$ & $28.58 \pm 0.97 c$ & $30.16 \pm 0.51 b$ & $26.75 \pm 0.41 \mathrm{~d}$ & $16.60 \pm 0.03 \mathrm{~h}$ & $23.20 \pm 1.08 \mathrm{f}$ & 0.301 & $<0.001$ \\
\hline Total MUFA & $53.92 \pm 0.29 \mathrm{a}$ & $35.45+1.84 \mathrm{c}$ & $10.09 \pm 0.10 \mathrm{~h}$ & $16.58+1.29 \mathrm{~g}$ & $28.06 \pm 0.10 \mathrm{e}$ & $25.76 \pm 0.02 \mathrm{f}$ & $46.27 \pm 0.05 \mathrm{~b}$ & $10.71+0.28 \mathrm{~h}$ & $30.47 \pm 0.67 \mathrm{~d}$ & 0.044 & $<0.001$ \\
\hline Total PUFA & $21.42 \pm 0.72 \mathrm{f}$ & $43.16 \pm 1.88 \mathrm{~d}$ & $64.79 \pm 0.81 \mathrm{~b}$ & $47.57 \pm 0.81 \mathrm{c}$ & $43.37 \pm 1.07 \mathrm{~d}$ & $44.08 \pm 0.53 \mathrm{~d}$ & $26.98 \pm 0.36 \mathrm{e}$ & $72.69 \pm 0.25 a$ & $46.33 \pm 0.40 \mathrm{c}$ & 0.341 & $<0.001$ \\
\hline
\end{tabular}

(C6:0) caproic acid; (C8:0) caprylic acid; (C10:0) capric acid; (C12:0) lauric acid; (C14:0) myristic acid; (C14:1) myristoleic acid; (C15:0) pentadecanoic acid; (C16:0) palmitic acid;

(C16:1) palmitoleic acid; (C17:0) heptadecanoic acid; (C18:0) stearic acid; (C18:1n9) oleic acid; (C18:2n6) linoleic acid; (C18:3n6) $\gamma$-linolenic acid; (C18:3n3) $\alpha$-linolenic acid; (C20:1) (C20:5n3) cis-5,811,14,17-e.0) arachidic acid, (C20:2) (C) acid. (SFA) Saturated Fatty Acids; (MUFA) Monounsaturated Fatty Acids; (PUFA) Polyunsaturated Fatty Acids. ${ }^{1}$ Homoscedasticity among mushroom samples was tested by the Levene test: homoscedasticity, $p>0.05$; heteroscedasticity, $p<0.05 .{ }^{2} p<0.05$ indicates that the mean value of at least one component differs from the others (in this case, multiple comparison tests were performed). $p$-Value $(n=81)$. For each mushroom sample, means within a column with different letters differ significantly $(p<0.05)$. nd $=$ not detected. 
Table 4. Organic acids composition (g/100 g of dry weight) of the studied wild mushrooms (mean \pm SD).

\begin{tabular}{ccccccc}
\hline Species & Oxalic Acid & Quinic Acid & Malic Acid & Citric Acid & Fumaric Acid $\begin{array}{c}\text { Total Identified } \\
\text { Organic Acids }\end{array}$ \\
\hline A. vitellinus & $0.18 \pm 0.01 \mathrm{~h}$ & nd & nd & nd & $0.03 \pm 0.01 \mathrm{~h}$ & $0.21 \pm 0.01 \mathrm{~h}$ \\
H. dusenii & $0.54 \pm 0.01 \mathrm{c}$ & nd & nd & nd & $0.31 \pm 0.01 \mathrm{~d}$ & $0.85 \pm 0.01 \mathrm{f}$ \\
C. magellanicus & $0.38 \pm 0.01 \mathrm{~d}$ & nd & $2.33 \pm 0.04 \mathrm{a}$ & $7.73 \pm 0.03 \mathrm{a}$ & $1.59 \pm 0.01 \mathrm{a}$ & $12.03 \pm 0.01 \mathrm{~b}$ \\
C. hariotii & $0.27 \pm 0.01 \mathrm{e}$ & nd & $0.35 \pm 0.01 \mathrm{e}$ & nd & $0.06 \pm .001 \mathrm{~g}$ & $0.68 \pm 0.01 \mathrm{~g}$ \\
F. antarctica & $0.24 \pm 0.01 \mathrm{f}$ & $0.08 \pm 0.01 \mathrm{~b}$ & $1.51 \pm 0.07 \mathrm{c}$ & nd & $0.51 \pm 0.03 \mathrm{c}$ & $2.34 \pm 0.07 \mathrm{~d}$ \\
F. endoxantha & $0.10 \pm 0.01 \mathrm{i}$ & nd & $1.01 \pm 0.01 \mathrm{~d}$ & nd & $0.30 \pm 0.01 \mathrm{~d}$ & $1.41 \pm 0.01 \mathrm{e}$ \\
G. gargal $^{\text {L. nuda }}$ & $0.22 \pm 0.01 \mathrm{~g}$ & nd & $1.91 \pm 0.01 \mathrm{~b}$ & nd & $0.19 \pm 0.01 \mathrm{f}$ & $2.32 \pm 0.01 \mathrm{~d}$ \\
R. patagonica & $2.22 \pm 0.02 \mathrm{~b}$ & $26.41 \pm 0.23 \mathrm{a}$ & nd & nd & $0.24 \pm 0.01 \mathrm{e}$ & $28.87 \pm 0.20 \mathrm{a}$ \\
\hline Homoscedasticity $^{1}$ & $2.53 \pm 0.02 \mathrm{a}$ & nd & nd & $7.48 \pm 0.04 \mathrm{~b}$ & $0.53 \pm 0.01 \mathrm{~b}$ & $10.54 \pm 0.01 \mathrm{c}$ \\
1-way ANOVA $^{2}$ & 0.036 & 0.005 & 0.006 & 0.006 & 0.122 & $<2$ \\
\hline
\end{tabular}

${ }^{1}$ Homoscedasticity among mushroom samples was tested by the Levene test: homoscedasticity, $p>0.05$; heteroscedasticity, $p<0.05 .{ }^{2} p<0.05$ indicates that the mean value of at least one component differs from the others (in this case, multiple comparison tests were performed). $p$-Value $(n=81)$. For each mushroom sample, means within a column with different letters differ significantly $(p<0.05)$. nd $=$ not detected.

Tocopherol composition differed between the studied species (Table 5). C. magellanicus presented significantly higher contents from both $\alpha$-tocopherol and $\beta$-tocopherol than the other species. The $\beta$-tocopherol was also present in significantly decreasing values in L. nuda, F. endoxantha and G. gargal. The $\alpha$-tocopherol, present in all species that presented tocopherols, showed minor proportions than $\beta$-tocopherol. Previous reports of tocopherols of L. nuda were detected and quantified, but using different methodologies and other units [13,34,59]. This work represents the first contribution in relation to the detection of tocopherols for the other eight studied species.

Table 5. Composition in tocopherols ( $\mu \mathrm{g} / 100 \mathrm{~g}$ of dry weight) of the studied wild mushrooms (mean $\pm \mathrm{SD}$ ).

\begin{tabular}{|c|c|c|c|}
\hline Species & $\alpha$-Tocopherol & $\beta$-Tocopherol & Total Tocopherols \\
\hline A. vitellinus & $8.09 \pm 0.42 b$ & nd & $8.09 \pm 0.42 \mathrm{e}$ \\
\hline H. dusenii & nd & nd & nd \\
\hline C. magellanicus & $30.65 \pm 0.84 a$ & $59.03 \pm 0.98 a$ & $89.68 \pm 0.14 a$ \\
\hline C. hariotii & $2.98 \pm 0.01 d$ & nd & $2.98 \pm 0.01 \mathrm{f}$ \\
\hline F. antarctica & $3.08 \pm 0.14 \mathrm{~d}$ & nd & $3.08 \pm 0.14 \mathrm{f}$ \\
\hline F. endoxantha & $5.13 \pm 0.28 c$ & $29.88 \pm 0.70 c$ & $35.01 \pm 0.98 c$ \\
\hline G. gargal & $2.99 \pm 0.28 \mathrm{~d}$ & $12.36 \pm 0.85 d$ & $15.35 \pm 1.13 d$ \\
\hline L. nuda & $1.99 \pm 0.28 \mathrm{e}$ & $36.25 \pm 0.98 b$ & $38.24 \pm 0.70 b$ \\
\hline R. patagonica & nd & nd & nd \\
\hline Homoscedasticity $^{1}$ & 0.069 & 0.048 & 0.058 \\
\hline 1-way ANOVA 2 & $<0.001$ & $<0.001$ & $<0.001$ \\
\hline
\end{tabular}

\footnotetext{
${ }^{1}$ Homoscedasticity among mushroom samples was tested by the Levene test: homoscedasticity, $p>0.05$; heteroscedasticity, $p<0.05 .^{2} p<0.05$ indicates that the mean value of at least one component differs from the others (in this case, multiple comparison tests were performed). $p$-Value $(n=81)$. For each mushroom sample, means within a column with different letters differ significantly $(p<0.05)$. nd $=$ not detected.
}

\subsection{Bioactive Compounds and Antioxidant Properties}

Three phenolic acids (galic, $p$-hydroxybenzoic and $p$-coumaric acids) and a related compound (cinnamic acid) were identified and quantified in some of the studied species (Table 6). Gallic acid was detected in a range between 1.26 and $4.56 \mu \mathrm{g} / 100 \mathrm{~g} \mathrm{dw}$, but was absent in H. dusenii, C. magellanicus and L. nuda. The lowest values of gallic acid were found in A. vitellinus, C. hariotii and G. gargal without statistical differences $(p<0.05)$. The $p$-hydroxybenzoic acid was only present in $F$. antarctica and R. patagonica, while $p$-coumaric acid was registered only in $R$. patagonica. This last species showed a noticeably higher phenolic acid concentration, mostly due to the contribution of $p$-hydroxybenzoic acid, compared with the other species. Barros et al. [50] working with Portuguese wild mushrooms also detected other species of the genus Ramaria (R. botritys) as the one with the highest phenolic acids 
concentration (356.7 mg/kg, dry matter). L. nuda did not show phenolic compounds at all in this study, although Barros et al. [50] reported the presence of $p$-hydroxybenzoic acid, $p$-coumaric acid and protocatechuic acid. These differences could be related to the mushroom origin and the edaphoclimatic conditions as well as stress conditions such as UV radiation, infection by pathogens and parasites, wounding, air pollution and exposure to extreme temperatures [50]. This is the first report concerning the phenolic acid composition of the other eight studied species.

Table 6. Composition in phenolic compounds of the studied wild mushrooms $(\mu \mathrm{g} / 100 \mathrm{~g} \mathrm{dw})(\mathrm{mean} \pm \mathrm{SD})$.

\begin{tabular}{|c|c|c|c|c|c|}
\hline Species & Gallic Acid & $p$-Hydroxybenzoic Acid & $p$-Coumaric Acid & Total & Cinnamic Acid \\
\hline A. vitellinus & $1.26 \pm 0.01 \mathrm{c}$ & $\operatorname{tr}$ & nd & $1.26 \pm 0.01 \mathrm{e}$ & nd \\
\hline H. dusenii & nd & $\operatorname{tr}$ & nd & nd & nd \\
\hline C. magellanicus & nd & nd & nd & nd & nd \\
\hline C. hariotii & $1.48 \pm 0.07 \mathrm{c}$ & nd & nd & $1.48 \pm 0.07 \mathrm{de}$ & nd \\
\hline F. antarctica & $3.14 \pm 0.05 b$ & $6.71 \pm 0.25 b$ & nd & $9.85 \pm 0.09 b$ & nd \\
\hline F. endoxhanta & $4.59 \pm 0.02 \mathrm{a}$ & nd & nd & $4.59 \pm 0.02 c$ & nd \\
\hline G. gargal & $1.70 \pm 0.01 \mathrm{c}$ & $\operatorname{tr}$ & nd & $1.70 \pm 0.01 \mathrm{~d}$ & nd \\
\hline L. nuda & nd & nd & nd & nd & nd \\
\hline R. patagonica & $4.56 \pm 0.06 a$ & $126.42 \pm 2.16 \mathrm{a}$ & $3.41 \pm 0.01$ & $134.39 \pm 2.10 \mathrm{a}$ & $3.10 \pm 0.01$ \\
\hline Homoscedasticity $^{1}$ & 0.008 & 0.008 & 0.007 & 0.013 & 0.007 \\
\hline 1-way ANOVA 2 & $<0.001$ & $<0.001$ & $<0.001$ & $<0.001$ & $<0.001$ \\
\hline
\end{tabular}

${ }^{1}$ Homoscedasticity among mushroom samples was tested by the Levene test: homoscedasticity, $p>0.05$; heteroscedasticity, $p<0.05{ }^{2} p<0.05$ indicates that the mean value of at least one component differs from the others (in this case, multiple comparison tests were performed). $p$-Value $(n=81)$. For each mushroom sample, means within a column with different letters differ significantly $(p<0.05)$. nd = not detected.

The composition in total phenolics and in vitro antioxidant activity of the studied wild mushrooms is shown in Table 7. R. patagonica presented the best results in all the antioxidant activity assays, with $\mathrm{EC}_{50}$ values of DPPH scavenging activity, reducing power, $\beta$-carotene bleaching inhibition and TBARS inhibition $\leq 1 \mathrm{mg} / \mathrm{mL}$, in agreement with its highest levels of total phenolics. In fact, it had been reported that the antioxidant activity of mushroom materials is well correlated with the content of phenolic compounds [57]. F. antarctica showed the lowest antioxidant activity ( $\mathrm{EC}_{50}$ values ranging from 0.95 to $13.8 \mathrm{mg} / \mathrm{mL}$ ) and low content of phenols (7.82 mg GAE/g extract), very close to those of C. hariotii, G. gargal and C. magellanicus.

The studied samples of L. nuda showed lower values of DPPH radical-scavenging activity, reducing power and $\beta$-carotene bleaching inhibition $(16.05,2.08$ and $12.24 \mathrm{mg} / \mathrm{mL}$, respectively) than those recorded by Pinto et al. [59], while TBARS inhibition value was similar $(5.80 \mathrm{mg} / \mathrm{mL})$ to that reported by Barros et al. [35]. Elmastas et al. [34] evaluate the antioxidant activity, L. nuda by applying a different methodology, while; Brujin et al. [44,45] did so for G. gargal, but using different solvents or heat treatment. Postemsky et al. [46], in a study of different species of Grifola. spp. on solid state fermentation to obtain biotransformed wheat grain, reported higher DPPH radical-scavenging values $(0.55 \mathrm{mg} / \mathrm{mL})$ and reducing power $(0.56 \mathrm{mg} / \mathrm{mL})$ activity for G. gargal than those detected in this work. 
Table 7. Total phenolics and in vitro antioxidant properties ( $\mathrm{EC}_{50}$ values) of the studied wild mushrooms (mean $\left.\pm \mathrm{SD}\right)$.

\begin{tabular}{|c|c|c|c|c|c|}
\hline Species & $\begin{array}{c}\text { Phenolics } \\
\text { (mg GAE/g extract) }\end{array}$ & $\begin{array}{l}\text { DPPH Scavenging } \\
\text { Activity }(\mathrm{mg} / \mathrm{mL})\end{array}$ & $\begin{array}{c}\text { Reducing Power } \\
(\mathrm{mg} / \mathrm{mL})\end{array}$ & $\begin{array}{c}\beta \text {-Carotene Bleaching } \\
\text { Inhibition }(\mathrm{mg} / \mathrm{mL})\end{array}$ & $\begin{array}{c}\text { TBARS Inhibition } \\
(\mathrm{mg} / \mathrm{mL})\end{array}$ \\
\hline A. vitellinus & $40.60 \pm 1.82 b$ & $17.05 \pm 0.34 b$ & $0.82 \pm 0.01 \mathrm{f}$ & $2.85 \pm 0.18 c$ & $0.38 \pm 0.02 \mathrm{de}$ \\
\hline H. dusenii & $16.40 \pm 0.49 \mathrm{e}$ & $17.88 \pm 0.52 b$ & $1.24 \pm 0.02 \mathrm{e}$ & $2.31 \pm 0.10 \mathrm{~d}$ & $1.33 \pm 0.15 b$ \\
\hline C. magellanicus & $9.86 \pm 0.46 f$ & $15.72 \pm 0.29 c$ & $3.77 \pm 0.03 a$ & $1.05 \pm 0.07 \mathrm{e}$ & $0.85 \pm 0.03 c$ \\
\hline C. hariotii & $8.48 \pm 0.38 \mathrm{~g}$ & $19.24 \pm 0.74 a$ & $2.17 \pm 0.03 c$ & $3.42 \pm 0.11 b$ & $0.14 \pm 0.01 \mathrm{ef}$ \\
\hline F. antarctica & $7.82 \pm 0.19 \mathrm{~g}$ & $13.78 \pm 0.18 \mathrm{~d}$ & $2.46 \pm 0.03 b$ & $2.94 \pm 0.08 c$ & $0.95 \pm 0.04 c$ \\
\hline F. endoxantha & $33.56 \pm 0.84 \mathrm{c}$ & $1.54 \pm 0.02 \mathrm{~g}$ & $0.79 \pm 0.01 \mathrm{~g}$ & $2.23 \pm 0.07 \mathrm{~d}$ & $0.44 \pm 0.06 \mathrm{~d}$ \\
\hline G. gargal & $9.77 \pm 0.23 f$ & $12.17 \pm 0.05 \mathrm{e}$ & $1.97 \pm 0.03 \mathrm{~d}$ & $3.31 \pm 0.16 b$ & $0.23 \pm 0.01 \mathrm{def}$ \\
\hline R. patagonica & $50.82 \pm 0.56 a$ & $0.77 \pm 0.04 \mathrm{~h}$ & $0.17 \pm 0.01 \mathrm{i}$ & $0.61 \pm 0.05 f$ & $0.06 \pm 0.01 \mathrm{f}$ \\
\hline Homoscedasticity $^{1}$ & $<0.001$ & $<0.001$ & $<0.001$ & $<0.001$ & $<0.001$ \\
\hline 1-way ANOVA 2 & $<0.001$ & $<0.001$ & $<0.001$ & $<0.001$ & $<0.001$ \\
\hline
\end{tabular}

${ }^{1}$ Homoscedasticity among mushroom samples was tested by the Levene test: homoscedasticity, $p>0.05$; heteroscedasticity, $p<0.05 .{ }^{2} p<0.05$ indicates that the mean value of at least one component differs from the others (in this case, multiple comparison tests were performed). $p$-Value $(n=81)$. For each mushroom sample, means within a column with different letters differ significantly $(p<0.05)$. 


\section{Materials and Methods}

\subsection{Collection and Identification of Fungi}

Specimens of nine species of wild edible mushrooms (Aleurodiscus vitellinus, Cortinarius magellanicus, Hydropus dusenii, Cyttaria hariotii, Fistulina antarctica, F. endoxantha, Grifola gargal, Lepista nuda and Ramaria patagonica) (Figure 1) were collected during fall and spring seasons of 2010, 2011 and 2012 in Nothofagus forest from National Parks of Chubut, Río Negro and Neuquén provinces, Argentina. The morphological identification of the collected specimens was performed according to previous reports [65-68]. Representative collections of each species was herborized and deposited in the Herbarium of the Patagonian Forest Research Center (CIEFAP; Esquel, Chubut, Argentina). Individual samples were pulverized and stored in freezer at $-18{ }^{\circ} \mathrm{C}$ in polyethylene bags for subsequent chemical analyses. Species analyses were performed on composite samples, including fruit bodies from collections from different stands.

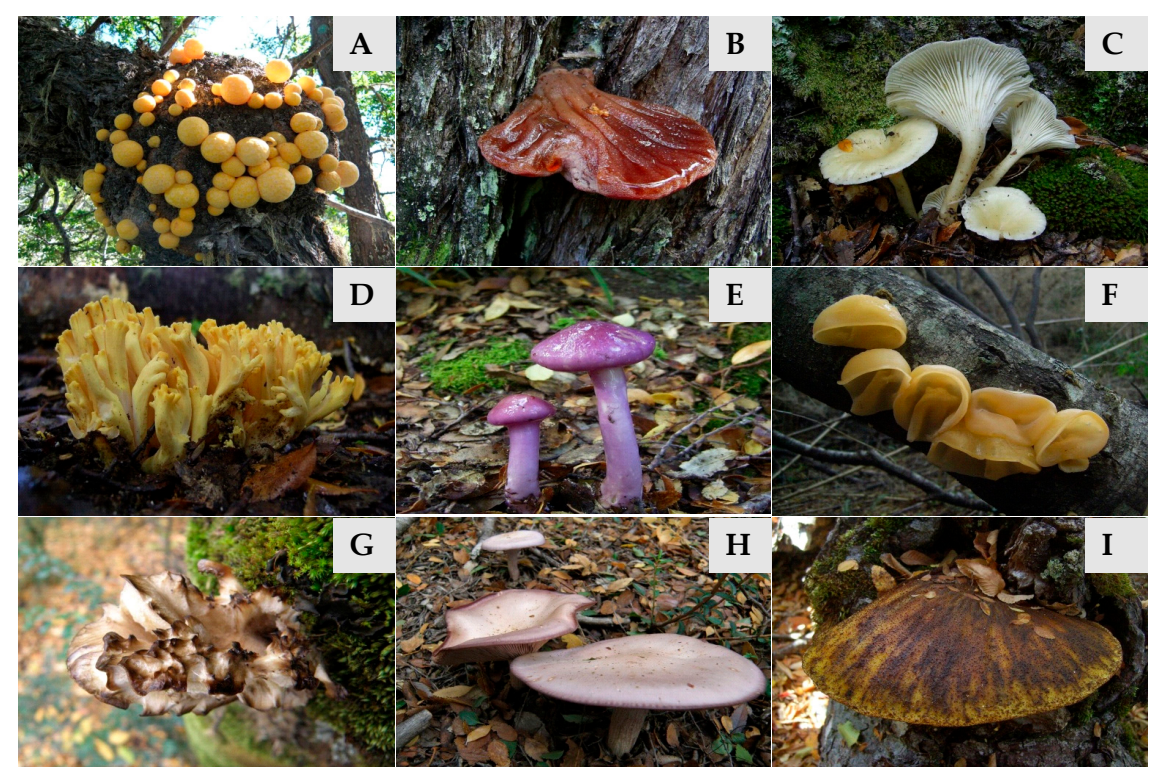

Figure 1. Frutifications of the wild studied mushrooms. (A) Cyttaria hariotii; (B) Fistulina antarctica; (C) Hydropus dusenii; (D) Ramaria patagonica; (E) Cortinarius magellanicus; (F) Aleurodiscus vitellinus;

(G) Grifola gargal; (H) Lepista nuda; (I) Fistulina endoxantha.

\subsection{Standards and Reagents}

Acetonitrile (99.9\%), n-hexane (97\%) and ethyl acetate $(99.8 \%)$ were of HPLC grade from Fisher Scientific (Lisbon, Portugal). The fatty acids methyl ester (FAME) reference standard mixture 37 (standard 47885-U) was purchased from Sigma (St. Louis, MO, USA), as also were other individual fatty acid isomers, L-ascorbic acid, trolox (6-hydroxy-2,5,7,8-tetramethylchroman-2-carboxylic acid), tocopherol, organic acid, phenolic acids and sugar standards. Racemic tocol, $50 \mathrm{mg} / \mathrm{mL}$, was purchased from Matreya (Pleasant Gap, PA, USA). 2,2-Diphenyl-1-picrylhydrazyl (DPPH) was obtained from Alfa Aesar (Ward Hill, MA, USA). Water was treated in a Milli-Q water purification system (TGI Pure Water Systems, Greenville, SC, USA).

\subsection{Determination of Nutritional and Chemical Composition}

\subsubsection{Macronutrients}

Mushroom samples were analyzed for chemical composition (protein, fat, carbohydrates and ash) using AOAC procedures [69]: Crude protein (AOAC 978.04), fat (AOAC 920.85), ash (AOAC 923.03) 
and total carbohydrates were calculated by difference. Results were expressed in $\mathrm{g}$ per $100 \mathrm{~g}$ of dry weight $(\mathrm{dw})$.

Energy was calculated according to the following equation: Energy $(\mathrm{Kcal})=4 \times(\mathrm{g}$ proteins $+\mathrm{g}$ carbohydrates) $+9 \times$ (g lipids) [12]. Results were expressed in kcal per $100 \mathrm{~g}$ of dry weight $(\mathrm{dw})$.

\subsubsection{Sugars Composition}

Free sugars were determined by high performance liquid chromatography coupled to a refraction index detector (HPLC-RI) based on the method used by Harada et al. [70] with minor modifications optimized by Heleno et al. [53]. Sugars were identified by comparing the relative retention times of sample peaks with standards and data was processed using a Clarity 2.4 Software (DataApex, Podohradska, Czech Republic). Quantification was performed using an internal standard (IS, raffinose) methodology and results were expressed in g per $100 \mathrm{~g}$ of dry weight.

\subsubsection{Fatty acids Composition}

Fatty acids (obtained after Soxhlet extraction) were determined by gas chromatography with flame ionization detection (GC-FID)/capillary column as described previously by the Heleno et al. [53] and after the following trans-esterification procedure. Fatty acids were processed using a Clarity 4.0.1.7 Software (DataApex, Podohradska, Czech Republic), identification was performed by comparing the relative retention times of FAME peaks from samples with standards and results were and expressed in relative percentage of each fatty acid.

\subsubsection{Organic Acids Composition}

Organic acids were determined by ultra-fast liquid chromatography coupled to a photo diode array detector (UFLC-PDA) following a procedure previously described by the authors [64]. The identification was performed by comparing relative retention times and UV spectra with commercial standards. Quantification was accessed by area comparison of the peaks recorded at $215 \mathrm{~nm}$ with calibration curves obtained from commercial standards and results were expressed in $\mathrm{g}$ per $100 \mathrm{~g}$ of dry weight.

\subsubsection{Tocopherol Composition}

Tocopherol content was determined following a procedure previously optimized and described by Heleno et al. [36], using a HPLC system (Smartline, Knauer, Germany), coupled to a fluorescence detector (FP-2020; Jasco, Japan) programmed for excitation at $290 \mathrm{~nm}$ and emission at $330 \mathrm{~nm}$. Data was processed using a Clarity 2.4 Software (DataApex). Compounds were identified by chromatographic comparisons with commercial standards and quantification was based on the fluorescence signal response, using an internal standard method. Tocopherol contents in mushrooms were expressed in $\mu \mathrm{g}$ per $100 \mathrm{~g}$ of dry mushroom.

\subsection{Evaluation of Bioactive Compounds and Antioxidant Properties}

\subsubsection{Extracts Preparation}

The extractions were performed using a fine dried powder $(20$ mesh; $2 \mathrm{~g}$ ) stirred with $30 \mathrm{~mL}$ of methanol at $25^{\circ} \mathrm{C}$ at $150 \mathrm{rpm}$ for $1 \mathrm{~h}$ and filtered through Whatman No. 4 paper. The residue was then re-extracted with one additional $30 \mathrm{~mL}$ portion of methanol. The combined methanolic extracts were evaporated at $35^{\circ} \mathrm{C}$ under reduced pressure (rotary evaporator Büchi R-210). The extracts were re-dissolved in methanol to a final concentration $20 \mathrm{mg} / \mathrm{mL}$ and were further diluted to different concentrations to be submitted to the following assays. 


\subsubsection{Phenolic Compounds Composition}

Total phenolics. Phenolics were estimated based on procedures previously described by Wolfe et al. [71]. Gallic acid was used to calculate the standard curve $\left(0.05-0.8 \mathrm{mM} ; \mathrm{Y}=1.8072 \mathrm{x}+0.0211 ; R^{2}=0.999\right)$ and results were expressed as $\mathrm{mg}$ of gallic acid equivalents (GAE) per $\mathrm{g}$ of extract.

Individual phenolic compounds. Phenolic compounds were determined by UFLC-PDA (Shimadzu Corporation, Kyoto, Japan) following a procedure previously described by the authors [72], with some modifications. The phenolic compounds were identified by comparing their retention time and UV-vis spectra with those obtained from commercial standards, when available. Quantification was obtained using calibration curves for each available phenolic standard and for the identified phenolic compounds for which a commercial standard was not available, quantification was based on calibration curves using compounds from the same phenolic group. Results were expressed in $\mu \mathrm{g}$ per $100 \mathrm{~g}$ of dry weight.

\subsubsection{Antioxidant Properties}

The antioxidant activity was evaluated by DPPH radical-scavenging activity, reducing power, inhibition of $\beta$-carotene bleaching in the presence of linoleic acid radicals and inhibition of lipid peroxidation using TBARS in brain homogenates $[7,57]$. The extract concentrations providing $50 \%$ of antioxidant activity or 0.5 of absorbance $\left(\mathrm{EC}_{50}\right)$ were calculated from the graphs of antioxidant activity percentages (DPPH, $\beta$-carotene bleaching and TBARS assays) or absorbance at $690 \mathrm{~nm}$ (reducing power assay) against extract concentrations. Trolox was used as standard.

\subsubsection{Statistical Analysis}

For mushroom species, three independent samples were analyzed and data was expressed as mean \pm standard deviation. All statistical tests were performed at a $5 \%$ significance level using IBM SPSS Statistics for Windows, version 22.0. (IBM Corp., Amonk, NY, USA). The fulfilment of the one-way ANOVA requirements, specifically the normal distribution of the residuals (data not shown) and the homogeneity of variance, was tested by means of the Shapiro Wilk's and the Levene's tests, respectively. All dependent variables were compared using Tukey's honestly significant difference (HSD) or Tamhane's T2 multiple comparison tests, when homoscedasticity was verified or not, respectively.

\section{Conclusions}

In conclusion, this study constitutes the first report on the chemical composition of A. vitellinus, $H$. dusenii, C. magellanicus, F. antarctica, F. endoxantha and R. patagonica. The studied species showed low fat values and high protein and carbohydrate contents, similar to what have been reported in previous studies involving wild edible mushrooms. Nevertheless, the high nutritional quality and unique organoleptic characteristics have to be documented in order to initiate the formal registration of many of these novel species in the Argentinian code of food. On the other hand, discovering new sources of nutraceuticals from these species put in value these non-timber forest products from Patagonia native forest and pose the challenge to work for a better management and conservation of this natural resource and habitats related to them. In particular, $R$. patagonica gave the highest antioxidant activity and the highest content of phenolic compounds.

Acknowledgments: The authors are grateful to the Agencia Nacional de Promoción Científica y Tecnológica FONCyT Argentina (grant PICT 2011-00118), CONICET (grant PIP 11220110100388), the Foundation for Science and Technology (FCT, Portugal) and FEDER under Program PT2020 for financial support to CIMO (UID/AGR/00690/2013), LSRE (Project UID/EQU/50020/2013) and L. Barros (SFRH/BPD/107855/2015) grant.

Author Contributions: Carolina Toledo and C. Barroetaveña designed the study, collected all the samples and leaded the manuscript writing. Ângela Fernandes, Lillian Barros and Isabel C. F. R. Ferreira performed all the chemical analysis, the statistics and participated in the manuscript writing.

Conflicts of Interest: The authors declare no conflict of interest. 


\section{References}

1. Boa, E. Wild edible fungi: A global overview of their use and importance to people. In Non-Wood Forest Products; FAO: Rome, Italy, 2004; pp. 1-147.

2. Dai, Y.C.; Yang, Z.L.; Cui, B.K.; Yu, C.J.; Zhou, L.W. Species diversity and utilization of medicinal mushrooms and fungi in China (Review). Int. J. Med. Mushrooms 2009, 11, 287-302. [CrossRef]

3. Chang, S.T.; Wasser, S.P. The role of culinary-medicinal mushrooms on human welfare with a pyramid model for human health. Int. J. Med. Mushrooms 2012, 14, 95-134. [CrossRef] [PubMed]

4. Wang, X.M.; Zhang, J.; Wu, L.H.; Zhao, Y.L.; Li, T.; Li, J.Q.; Wang, Y.Z.; Liu, H.G. A mini-review of chemical composition and nutritional value of edible wild-grown mushroom from China. Food Chem. 2014, 151, 279-285. [CrossRef] [PubMed]

5. Villarreal, L.; Pérez-Moreno, J. Los hongos comestibles silvestres de México, un enfoque integral. Micol. Neotrop. Apl. 1989, 2, 77-114.

6. Chang, S.T.; Miles, P.G. The nutritional attributes of edible mushrooms. In Mushrooms: Cultivation, Nutritional Value, Medicinal Effect and Environmental Impact, 2nd ed.; CRC Press: Boca Raton, FL, USA, 2004; pp. 27-52.

7. Barros, L.; Baptista, P.; Correia, D.M.; Morais, J.S.; Ferreira, I.C.F.R. Effects of conservation treatment and cooking on the chemical composition and antioxidant activity of Portuguese wild edible mushrooms. J. Agric. Food Chem. 2007, 55, 4781-4788. [CrossRef] [PubMed]

8. Kalač, P. Chemical composition and nutritional value of European species of wild growing mushrooms: A review. Food Chem. 2009, 113, 9-16. [CrossRef]

9. Kalač, P. A review of chemical composition and nutritional value of wild-growing and cultivated mushrooms. J. Sci. Food Agric. 2013, 93, 209-218. [CrossRef] [PubMed]

10. Kanu, P.J.; Zhu, K.; Kanu, J.B.; Zhou, H.; Qian, H.; Zhu, K. Biologically active components and nutraceuticals in sesame and related products: A review and prospect. Trends Food Sci. Technol. 2007, 18, 599-608. [CrossRef]

11. Díez, V.A.; Alvarez, A. Compositional and nutritional studies on two wild edible mushrooms from northwest Spain. Food Chem. 2001, 75, 417-422. [CrossRef]

12. Manzi, P.; Marconi, S.; Aguzzi, A.; Pizzoferrato, L. Commercial mushrooms: Nutritional quality and effect of cooking. Food Chem. 2004, 84, 201-206. [CrossRef]

13. Barros, L.; Venturini, B.A.; Baptista, P.; Estevinho, L.M.; Ferreira, I.C.F.R. Chemical composition and biological properties of Portuguese wild mushrooms: A comprehensive study. J. Agric. Food Chem. 2008, 56, 3856-3862. [CrossRef] [PubMed]

14. Reis, F.S.; Barros, L.; Martins, A.; Ferreira, I.C.F.R. Chemical composition and nutritional value of the most widely appreciated cultivated mushrooms: An inter-species comparative study. Food Chem. Toxicol. 2012, 50, 191-197. [CrossRef] [PubMed]

15. Ouzouni, P.K.; Riganakos, K.A. Nutritional value and metal content profile of Greek wild edible fungi. Acta Aliment. 2007, 36, 99-110. [CrossRef]

16. Ouzouni, P.K.; Petridis, D.; Koller, W.D.; Riganakos, K.A. Nutritional value and metal content of wild edible mushrooms collected from West Macedonia and Epirus, Greece. Food Chem. 2009, 115, 1575-1580. [CrossRef]

17. Çağlarirmak, N. The nutrients of exotic mushrooms (Lentinula edodes and Pleurotus species) and an estimated approach to the volatile compounds. Food Chem. 2007, 105, 1188-1194. [CrossRef]

18. Agahar-Murugkar, D.; Subbulakshmi, G. Nutritional value of edible wild mushrooms collected from the Khasi hills of Meghalaya. Food Chem. 2005, 89, 599-603. [CrossRef]

19. Olfati, J.; Peyvast, G.; Mami, Y. Identification and chemical properties of popular wild edible mushrooms from northern Iran. J. Hortic. For. 2009, 1, 48-51.

20. Sanmee, R.; Dell, B.; Lumyong, P.; Izumori, K.; Lumyong, S. Nutritive value of popular wild edible mushrooms from northern Thailand. Food Chem. 2003, 82, 527-532. [CrossRef]

21. Stojković, D.; Reis, F.S.; Ferreira, I.C.F.R.; Barros, L.; Glamočlija, J.; Ćirić, A.; Nikolić, M.; Stević, T.; Giveli, A.; Soković, M. Tirmania pinoyi: Chemical composition, in vitro antioxidant and antibacterial activities and in situ control of Staphylococcus aureus in chicken soup. Food Res. Int. 2013, 53, 56-62. [CrossRef]

22. Furlani, R.P.Z.; Godoy, H.T. Valor nutricional de cogumelos comestíveis. Ciênc. Tecnol. Aliment. 2007, 27, 154-157. [CrossRef] 
23. León-Guzmán, M.F.; Silva, I.; López, M.G. Proximate Chemical Composition, Free Amino Acid Contents and Free Fatty Acid Contents of Some Wild Edible Mushrooms from Querétaro, México. Food Chem. 1997, 45, 4329-4332. [CrossRef]

24. Arce, S.; Cerutti, S.; Olsina, R.; Gomez, M.R.; Martinez, L.D. Trace element profile of a wild edible mushroom (Suillus granulatus). J. AOAC Int. 2008, 91, 853-857. [PubMed]

25. Omarini, A.; Henning, C.; Ringuelet, J.; Zygadlo, J.A.; Albertó, E. Volatile composition and nutritional quality of the edible-mushroom Polyporus tenuiculus grown on different agro-industrial waste. Int. J. Food Sci. Technol. 2010, 45, 1603-1609. [CrossRef]

26. Di Anibal, C.; Farenzena, S.; Rodríguez, M.S.; Albertengo, L. Chemical composition and nutritional value of Argentine commercial edible mushrooms. J. Verbrauch. Lebensm. 2015, 10, 155-164. [CrossRef]

27. Abuajah, C.I.; Ogbonna, A.C.; Osuji, C.M. Functional components and medicinal properties of food: A review. J. Food Sci. Technol. 2015, 52, 2522-2529. [CrossRef] [PubMed]

28. Younesi, E.; Ayseli, M.T. An integrated systems-based model for substantiation of health claims in functional food development. Trends Food Sci. Technol. 2015, 41, 95-100. [CrossRef]

29. Wildman, R.E.; Kelley, M. Nutraceuticals and functional foods. In Handbook of Nutraceuticals and Functional Foods, 4th ed.; Wildman, R.E., Wildman, R., Wallace, T.C., Eds.; CRC Press: Boca Raton, FL, USA, 2016; pp. 1-22.

30. Lindequist, U.; Niedermeyer, T.H.J.; Jülich, W.D. The pharmacological potential of mushrooms. Evid. Based Complement. Altern. Med. 2005, 2, 285-299. [CrossRef] [PubMed]

31. Roselló-Soto, E.; Parniakov, O.; Deng, Q.; Patras, A.; Koubaa, M.; Grimi, N.; Boussetta, N.; Tiwari, B.K.; Vorobiev, E.; Lebovka, N.; et al. Application of non-conventional extraction methods: Toward a sustainable and green production of valuable compounds from mushrooms. Food Eng. Rev. 2016, 8, 214-234. [CrossRef]

32. Mau, J.L.; Lin, H.C.; Song, S.F. Antioxidant properties of several speciality mushrooms. Food Res. Int. 2002, 35, 519-526. [CrossRef]

33. Lo, K.M.; Cheung, P.C.K. Antioxidant activity of extracts from the fruiting bodies of Agrocybe aegerita var. alba. Food Chem. 2005, 89, 533-539. [CrossRef]

34. Elmastas, M.; Isildak, O.; Turkekul, I.; Temur, N. Determination of antioxidant activity and antioxidant compounds in wild edible mushrooms. J. Food Compos. Anal. 2007, 20, 337-345. [CrossRef]

35. Barros, L.; Cruz, T.; Baptista, P.; Estevinho, L.M.; Ferreira, I.C.F.R. Wild and commercial mushrooms as source of nutrients and nutraceuticals. Food Chem. Toxicol. 2008, 46, 2742-2747. [CrossRef] [PubMed]

36. Heleno, S.A.; Barros, L.; Sousa, M.J.; Martins, A.; Ferreira, I.C.F.R. Tocopherols composition of Portuguese wild mushrooms with antioxidant capacity. Food Chem. 2010, 119, 1443-1450. [CrossRef]

37. Heleno, S.A.; Barros, L.; Martins, A.; Queiroz, M.J.R.P.; Morales, P.; Fernández-Ruiz, V.; Ferreira, I.C.F.R. Chemical composition, antioxidant activity and bioaccessibility studies in phenolic extracts of two Hericium wild edible species. Food Sci. Technol. 2015, 63, 475-481. [CrossRef]

38. Reis, F.S.; Heleno, S.A.; Barros, L.; Sousa, M.J.; Martins, A.; Santos-Buelga, C.; Ferreira, I.C.F.R. Toward the antioxidant and chemical characterization of mycorrhizal mushrooms from Northeast Portugal. J. Food Sci. 2011, 76, 824-830. [CrossRef] [PubMed]

39. Gamundí, I.J.; Horak, E. Hongos de los Bosques Andino-Patagónicos; Vazquez Mazzini Editores: Buenos Aires, Argentina, 1993; pp. 1-144.

40. Toledo, C.; Barroetaveña, C.; Rajchenberg, M. Hongos Comestibles Silvestres de la región Andino Patagónica de la Argentina; Centro Forestal CIEFAP: Esquel, Argentina, 2016; pp. 1-71.

41. Gamundí, I.J.; Muchnik de Lederkremer, R.M. Los hongos andino-patagónicos del género Cyttaria. Sus hidratos de carbono. Cienc. Investig. 1989, 43, 4-13.

42. Schmeda-Hirschmann, G.; Razmilic, I.; Reyes, S.; Gutierrez, M.I.; Loyola, J.I. Biological activity and food analysis of Cyttaria spp. (Discomycetes). Econ. Bot. 1999, 53, 30-40. [CrossRef]

43. Schmeda-Hirschmann, G.; Razmilic, I.; Gutierrez, M.I.; Loyola, J.I. Proximate Composition and biological activity of food plants gathered by Chilean Amerindians. Econ. Bot. 1999, 53, 177-187. [CrossRef]

44. Bruijn, J.; Loyola, C.; Aqueveque, P.; Cañumir, J.; Cortéz, M.; France, A. Influence of heat treatment on the antioxidant properties of Grifola gargal hydro-alcoholic extracts. Micol. Apl. Int. 2008, 20, 27-34.

45. Bruijn, J.; Loyola, C.; Aqueveque, P.; Cañumir, J.; Cortéz, M.; France, A. Antioxidant properties of extracts obtained from Grifola gargal mushrooms. Micol. Apl. Int. 2009, 21, 11-18. 
46. Postemsky, P.D.; Curvetto, N.R. Enhancement of wheat grain antioxidant activity by Solid-state fermentation with Grifola spp. J. Med. Food 2014, 17, 543-549. [CrossRef] [PubMed]

47. Postemsky, P.D.; Curvetto, N.R. Submerged culture of Grifola gargal and G. sordulenta (higher basidiomycetes) from Argentina as a source of mycelia with antioxidant activity. Int. J. Med. Sci. Mushrooms 2015, 17, 65-76. [CrossRef]

48. Harada, E.; D'Alessandro-Gabazza, C.N.; Toda, M.; Morizono, T.; Chelakkot-Govindalayathil, A.L.; Roeen, Z.; Urawa, M.; Yasuma, T.; Yano, Y.; Sumiya, T.; et al. Amelioration of Atherosclerosis by the New Medicinal Mushroom Grifola gargal Singer. J. Med. Food 2015, 18, 872-881. [CrossRef] [PubMed]

49. Colak, A.; Kolcuoglu, Y.; Sesli, E.; Dalmar, O. Biochemical Composition of Some Turkish Fungi. Asian J. Chem. 2007, 19, 2193-2199.

50. Barros, L.; Dueñas, M.; Ferreira, I.C.F.R.; Baptista, P.; Santos-Buelga, C. Phenolic acids determination by HPLC-DAD-ESI/MS in sixteen different Portuguese wild mushrooms species. Food Chem. Toxicol. 2009, 47, 1076-1079. [CrossRef] [PubMed]

51. Colak, A.; Faiz, O.; Sesli, E. Nutritional composition of some wild edible mushrooms. Turk. J. Biochem. 2009, 34, 25-31.

52. Pereira, E.; Barros, L.; Martins, A.; Ferreira, I.C.F.R. Towards chemical and nutritional inventory of Portuguese wild edible mushrooms in different habitats. Food Chem. 2012, 130, 394-403. [CrossRef]

53. Heleno, S.A.; Barros, L.; Sousa, M.J.; Martins, A.; Ferreira, I.C.F.R. Study and characterization of selected nutrients in wild mushrooms from Portugal by gas chromatography and high performance liquid chromatography. Microchem. J. 2009, 93, 195-199. [CrossRef]

54. Flegg, P.B.; Maw, G. Mushrooms and their possible contribution to world protein needs. Mushroom J. 1977, 48, 395-403.

55. Breene, W.M. Nutritional and medicinal value of specially mushrooms. J. Food Protect. 1990, 53, 883-894.

56. Yilmaz, N.; Solmaz, M.; Türkekul, İ.; Elmastaş, M. Fatty acid composition in some wild edible mushrooms growing in the middle Black Sea region of Turkey. Food Chem. 2006, 99, 168-174. [CrossRef]

57. Barros, L.; Baptista, P.; Correia, D.; Casal, S.; Oliveira, B.; Ferreira, I.C.F.R. Fatty acid and sugar compositions and nutritional value of five edible mushrooms from Northeast Portugal. Food Chem. 2007, 105, 140-145. [CrossRef]

58. Ribeiro, B.; de Pinho, P.G.; Andrade, P.B.; Baptista, P.; Valentão, P. Fatty acid composition of wild edible mushrooms species: A comparative study. Microchem. J. 2009, 93, 29-35. [CrossRef]

59. Pinto, S.; Barros, L.; Sousa, M.J.; Ferreira, I.C.F.R. Chemical characterization and antioxidant properties of Lepista nuda fruiting bodies and mycelia obtained by in vitro culture: effects of collection habitat and culture media. Food Res. Int. 2013, 51, 496-502. [CrossRef]

60. Puiggrós, C.; Chacón, P.; Armadans, L.I.; Clapés, J.; Planas, M. Effects of oleic-rich andomega-3-rich diets on serum lipid pattern and lipid oxidation in mildly hypercholesterolemic patients. Clin. Nutr. 2002, 21, 79-87. [CrossRef] [PubMed]

61. Pacheco, Y.M.; López, S.; Bermúdez, B.; Abia, R.; Villar, J.; Muriana, F.J.G. A meal rich in oleic acid beneficially modulates postprandial sICAM-1 and sVCAM-1 in normotensive and hypertensive hypertriglyceridemic subjects. J. Nutr. Biochem. 2008, 19, 200-205. [CrossRef] [PubMed]

62. Voet, D.; Voet, J.G. Biochemistry, 3rd ed.; John Wiley \& Sons: New York, NY, USA, 2004; pp. 1-613.

63. Ribeiro, B.; Lopes, R.; Andrade, P.B.; Seabra, R.M.; Gonçalves, R.F.; Baptista, P.; Quelhas, I. Comparative study of phytochemicals and antioxidant potential of wild edible mushroom caps and stipes. Food Chem. 2008, 110, 47-56. [CrossRef] [PubMed]

64. Barros, L.; Pereira, C.; Ferreira, I.C.F.R. Optimized analysis of organic acids in edible mushrooms from Portugal by ultra fast liquid chromatography and photodiode array detection. Food Anal. Methods 2013, 6, 309-316. [CrossRef]

65. Singer, R. Mycoflora australis. Beihefte zur Nova Hedwigia 1969, 29, 1-405.

66. Horak, E. Fungi, Basidiomycetes, Agaricales y Gasteromycetes Secotioides; Consejo Nacional de Investigaciones Científicas y Técnicas: Buenos Aires, Argentina, 1979; pp. 1-525.

67. Moser, M.; Horak, E. Cortinarius Fr. und nahe Verwandte Gattungen in Sudamerika; Beiheft zur Nova. Hedwigia; J. Cramer: Vaduz, Germany, 1975; pp. 1-628. 
68. Valenzuela-Flores, E. Estudio Sistemático, Corológico y Ecológico de los Agaricales Sensu Lato de los Bosques Autóctonos de la Región de los Lago en Chile. Ph.D. Thesis, Universidad de Alcalá de Henares, Facultad de Ciencias, Spain, 1993.

69. AOAC. Official Methods of Analysis, 16th ed.; Association of Official Analytical Chemists: Arlington, VA, USA, 1995.

70. Harada, A.; Gisusi, S.; Yoneyama, S.; Aoyama, M. Effects of strain and cultivation medium on the chemical composition of the taste components in fruit-body of Hypsizygus marmoreus. Food Chem. 2004, 84, 265-270. [CrossRef]

71. Wolfe, K.; Wu, X.; Liu, R.H. Antioxidant activity of apple peels. J. Agric. Food Chem. 2003, 51, 609-614. [CrossRef] [PubMed]

72. Vaz, J.A.; Barros, L.; Martins, A.; Santos-Buelga, C.; Vasconcelos, M.H.; Ferreira, I.C.F.R. Chemical composition of wild edible mushrooms and antioxidant properties of their water soluble polysaccharidic and ethanolic fractions. Food Chem. 2011, 126, 610-616. [CrossRef]

Sample Availability: Not available.

(C) 2016 by the authors; licensee MDPI, Basel, Switzerland. This article is an open access article distributed under the terms and conditions of the Creative Commons Attribution (CC-BY) license (http://creativecommons.org/licenses/by/4.0/). 